\title{
Salmonella Serotypes Associated with Illnesses after Thanksgiving Holiday, United States, 1998-2018
}

Farrell A. Tobolowsky, Zhaohui Cui, Robert M. Hoekstra, Beau B. Bruce

We sought to determine which Salmonella serotypes cause illness related to the Thanksgiving holiday in the United States and to foods disproportionately eaten then (e.g., turkey). Using routine surveillance for 1998-2018 and a case-crossover design, we found serotype Reading to be most strongly associated with Thanksgiving.

Thanksgiving, celebrated annually in the United 1 States on the fourth Thursday of November, often brings together family and friends who eat specific traditional foods, such as mashed potatoes, cranberry sauce, and pumpkin pie; the most prominent food eaten is turkey (1). In 2017, $\approx 45$ million turkeys were produced for Thanksgiving, $\approx 18 \%$ of annual production (2). Turkey is popular across regions, races, sexes, and generations; $88 \%$ of persons in the United States report eating turkey during their Thanksgiving meal $(1,3,4)$.

Foodborne Salmonella infections cause substantial illness and death in the United States: an estimated 1 million cases, 20,000 hospitalizations, and 400 deaths occur annually (5). Typical illness consists of diarrhea, fever, and abdominal pain lasting 3-7 days; only a minority of persons seek health care. Incubation typically ranges from 6 hours to 6 days (5). Salmonella outbreaks caused by serotypes Hadar and Saint Paul have been most commonly attributed to turkey, and serotypes Enteritidis, Heidelberg, and Typhimurium have been frequent causes of turkey-associated outbreaks (6). During 2015-2020, Reading and Hadar were the serotypes most often isolated from turkeys (7); less is known about which serotypes cause turkey-associated sporadic Salmonella infections. We aimed to determine which Salmonella serotypes cause sporadic enteric infections after the Thanksgiving

Author affiliation: Centers for Disease Control and Prevention,

Atlanta, Georgia, USA

DOI: https://doi.org/10.3201/eid2801.211986 holiday and are most likely related to foods disproportionately eaten then, particularly turkey.

\section{The Study}

The Laboratory-based Enteric Disease Surveillance (LEDS) system captures enteric infections with Salmonella species in the United States through passive surveillance of laboratory-confirmed isolates. State and territorial public health laboratories serotype Salmonella isolates; any unusual serotypes are sent to the Centers for Disease Control and Prevention's National Salmonella Reference Laboratory (National Center for Emerging and Zoonotic Infectious Diseases, Division of Foodborne, Waterborne, and Environmental Diseases, Enteric Diseases Laboratory Branch) for further characterization. This surveillance system obtains demographic information; specimen source; collection date; test result; serotype; and, if available, outbreak association. We included infections that had fully serotyped Salmonella isolates that occurred from 1998 through 2018 and excluded isolates confirmed to be outbreak-associated.

Using a case-crossover design, we determined a case window for each year using the date of Thanksgiving, a minimum incubation period, and a window length. To account for seasonal variation in infections, we created nonexposure case windows before and after Thanksgiving by using the same case window length with a washout period separating the exposure and nonexposure windows (Appendix Figure, https:// wwwnc.cdc.gov/EID/article/28/1/211986-App1.pdf). Our primary analysis used a 7-day case window after a minimum 2-day incubation period after Thanksgiving, with a 5-day washout. Control windows were also 7 days.

We conducted sensitivity analyses evaluating different minimum incubations, case windows, and washouts and stratified all analyses by serotype. To account for reporting biases in Salmonella cases 
during holidays and nonholidays, we used all Salmonella cases associated with serotypes other than the serotype under consideration as the comparison group. We calculated odds ratios (ORs) for the entire study period and compared ORs for 1998-2007 with those for 2008-2018. We conducted descriptive demographic and clinical analyses with SAS versions 9.4 (https:/ / www.sas.com) and calculated ORs and unadjusted $p$ values with $R$ version 3.6.1 (R Foundation for Statistical Computing, https:// www.r-project.org) using unconditional maximumlikelihood estimation with 95\% CIs from the normal approximation.

Among 846,449 patients reported during 19982018 (Appendix Table 1), median patient age was 27 years; $52.5 \%$ of patients were female, and $82.2 \%$ were white. Isolates were identified by culture $(n=823,793$ [99.8\%]) and culture-independent diagnostic testing $(\mathrm{n}=1,919[0.2 \%])$. Specimens were most commonly obtained from stool $(86.8 \%)$, urine $(6.3 \%)$, and blood $(6.3 \%)$. The most frequent serotypes were Enteritidis (142,687 [18.3\%]), Typhimurium (131,216 [16.8\%]), and Newport (82,155 [10.5\%]).

In our primary analysis, serotype Reading had the highest OR of association with the Thanksgiving holiday $(2.18,95 \%$ CI 1.58-3.01; p<0.0001) (Figure). Other serotypes with significantly increased ORs were Baildon (OR 1.92, 95\% CI 1.04-3.52; $\mathrm{p}=$ 0.03), Worthington (OR 1.87, 95\% CI 1.06-3.3; $\mathrm{p}=$ 0.03 ), Ohio (OR 1.74, 95\% CI 1.06-2.86; $\mathrm{p}=0.03$ ), Hadar (OR 1.66, 95\% CI 1.35-2.05; p<0.0001), Derby (OR 1.57, 95\% CI 1.2-2.06; $\mathrm{p}=0.001$ ), Brandenburg (OR 1.42, 95\% CI 1.01-2.01; p = 0.045), Schwarzengrund (OR 1.40, 95\% CI 1.06-1.86; $\mathrm{p}=0.02$ ), 4,[5],12:i- (OR 1.39, 95\% CI 1.25-1.54; $\mathrm{p}<0.0001)$, and Heidelberg (OR 1.34, 95\% CI 1.21-1.49; p<0.0001).
We found no other significant positive associations among serotypes with $>50$ patients who became ill during the 2-9 days after Thanksgiving.

In sensitivity analyses, case windows with varying durations of minimum incubation (range 0-12 days), illness (range 5-7 days), and washout (range 0-5 days) produced similar sets of serotypes with significantly increased ORs, most commonly Reading, Hadar, 4,[5],12:i-, Derby, Heidelberg, and Schwarzengrund (Appendix Table 2). Comparing 1998-2007 with 2008-2018, ORs for Baildon, Derby, Hadar, 4,[5],12:i:-, and Ohio increased by $\geq 45 \%$ from the first to the second period, but significantly so only for 4,[5], 12:i:-. Three more serotypes had significant ORs in only 1 period (1998-2007, Javiana OR 1.23, Mbandaka OR 1.62; 2008-2018, Infantis OR 1.21; Appendix Table 3).

\section{Conclusions}

Salmonella Reading was the serotype most strongly associated with illness during the Thanksgiving holiday. Given the dramatic increase in turkey consumption around Thanksgiving, one might expect that serotypes we identified are primarily associated with turkey consumption, and indeed, Reading caused a multistate outbreak with a raw turkey source during 2017-2019 (8), and a new clone of this serotype has emerged since 2014 in commercial turkey production (9). Other serotypes significantly associated with Thanksgiving in our study (i.e., Hadar, Schwarzengrund, and Heidelberg) have also been associated with turkey $(6,10)$.

Other significantly associated serotypes are not among those most commonly identified in turkey (e.g., Heidelberg and 4,[5],12:i- are more commonly identified in chicken; Derby, Brandenburg,
Figure. ORs for Salmonella serotypes associated with the Thanksgiving holiday, United States, 1998-2018. Error bars indicate $95 \% \mathrm{Cls}$. No significant positive associations with Thanksgiving were found among other serotypes with $>50$ patients who became ill during 2-9 days after Thanksgiving (i.e., Litchfield, Braenderup, Infantis, Senftenberg, Anatum, Dublin, Mbandaka, Typhimurium, and Javiana.) Serotypes with $>50$ patients inversely associated with Thanksgiving and significant p values include Poona (OR 0.77), Panama (OR 0.71), Newport (OR 0.93), and Paratyphi B var. L(+) tartrate+ (OR 0.75). These serotypes probably are associated with foods not eaten more frequently on Thanksgiving or other exposures not more frequently experienced on Thanksgiving than other times of the year. OR, odds ratio.

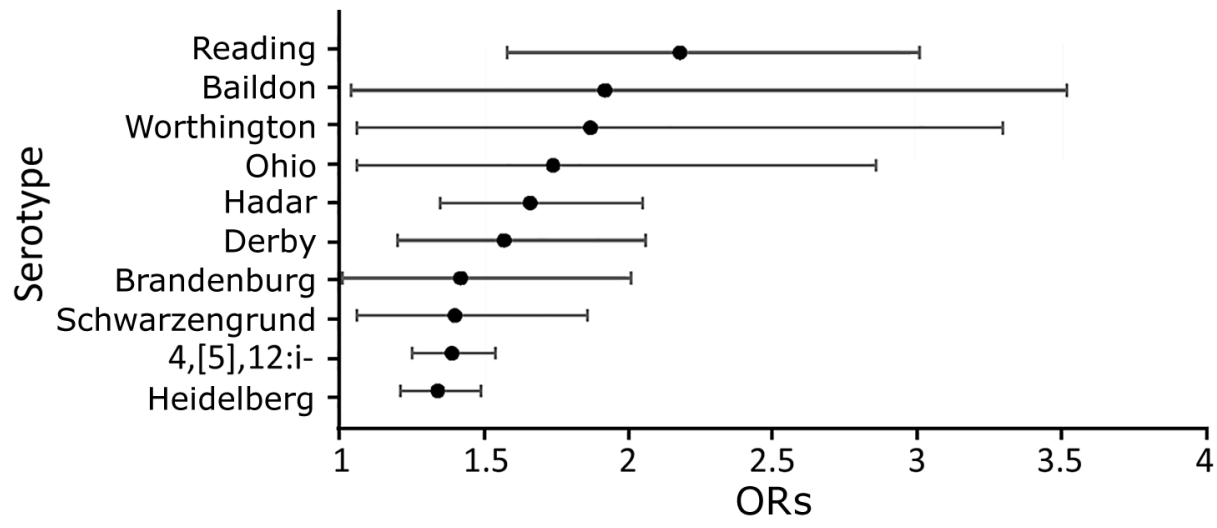


and 4,[5],12:i- in swine and pork; and 4,[5],12:i- in cattle). However, all these serotypes have been found in turkeys and in retail samples of turkey or have been associated with outbreaks attributed to turkey (11-15). Some of the serotypes significantly increased after Thanksgiving, such as, Baildon and Ohio, were rare, causing <200 illnesses annually, and were not reported among food animals, retail products, or outbreaks during 2015-2020 (7). Although our study may have identified serotypes associated with other foods eaten during the Thanksgiving holiday, particular attention probably should be paid to evidence of these serotypes emerging in turkey production.

The first limitation of our study is that LEDS is a passive surveillance system and does not capture mild or asymptomatic infections for which ill persons do not seek healthcare or submit a specimen. Although we removed cases reported as outbreakassociated, those data are not reported by all states, and some outbreak-associated cases most likely are included. Missing data in LEDS varies, but serotype and date are largely complete. Although sensitivity analyses demonstrate consistency across time windows, misalignment of windows with causative exposures could have resulted in biases, possibly differential, from, for example, differences in healthcare seeking because of the holiday itself. Our study may be subject to ecologic bias because individual food exposures are unknown. We did not adjust for multiple testing because this analysis is intended to be hypothesis-generating rather than confirmatory.

Our case-crossover approach could be helpful for other pathogens and their subtypes that are likely to cause illnesses from a certain food disproportionately eaten during a brief period, such as turkey during the Thanksgiving holiday. Our technique provides unique insights into the causes of sporadic illness throughout the year and their changes over multiyear periods using no more than routine surveillance data and may provide valuable information to industry, regulators, and public health officials that could help guide monitoring and interventions to prevent illnesses and their associated morbidity and mortality. Consumers can also help protect themselves from Salmonella by following the 4 steps to food safety (https://www.cdc.gov/ foodsafety/keep-food-safe.html).

\section{Acknowledgments}

We thank Patricia Griffin for her innovative ideas and helpful feedback on our manuscript.

\section{About the Author}

Dr. Tobolowsky is an Epidemic Intelligence Service Officer in the Division of Foodborne, Waterborne, and Environmental Diseases, National Center for Emerging and Zoonotic Infectious Diseases, Centers for Disease Control and Prevention. Her primary research interests include the epidemiology of and outbreak response for communicable diseases of public health importance.

\section{References}

1. History Channel. Thanksgiving 2021 [cited 2021 May 17]. http:/ / www.history.com/topics/thanksgiving/history-ofthanksgiving

2. Calfas J. Here's how many turkeys are killed each year for Thanksgiving. Time. 2017 Nov 16 [cited 2021 Jun 22]. https:/ / time.com/5022315/turkeys-killed-thanksgiving

3. Sifton S. The American Thanksgiving. New York Times. 2016 Nov 15 [cited 2021 Jun 22]. https:/ / www.nytimes.com/ interactive/2016/11/16/dining/thanksgiving-dinner-inamerica.html

4. Wells-Barrett C. Turkeys are estimated to cost Americans \$1.1 billion for Thanksgiving 2020 [cited 2021 Jun 11]. https://www.finder.com/american-thanksgivingturkey-spend

5. Eikmeier D, Medus C, Smith K. Incubation period for outbreak-associated, non-typhoidal salmonellosis cases, Minnesota, 2000-2015. Epidemiol Infect. 2018;146:423-9. https://doi.org/10.1017/S0950268818000079

6. Jackson BR, Griffin PM, Cole D, Walsh KA, Chai SJ. Outbreak-associated Salmonella enterica serotypes and food commodities, United States, 1998-2008. Emerg Infect Dis. 2013;19:1239-44. https:// doi.org/10.3201/eid1908.121511

7. US Food Safety and Inspection Service, US Department of Agriculture. Raw comminuted turkey sampling. 2020 Dec 26 [cited 2021 Jun 22]. https:/ / www.fsis.usda.gov/ news-events/publications/raw-comminuted-turkeysampling

8. Hassan R, Buuck S, Noveroske D, Medus C, Sorenson A, Laurent J, et al. Multistate outbreak of Salmonella infections linked to raw turkey products - United States, 2017-2019. MMWR Morb Mortal Wkly Rep. 2019;68:1045-9. https://doi.org/10.15585/mmwr.mm6846a1

9. Miller EA, Elnekave E, Flores-Figueroa C, Johnson A, Kearney A, Munoz-Aguayo J, et al. Emergence of a novel Salmonella enterica serotype Reading clonal group is linked to its expansion in commercial turkey production, resulting in unanticipated human illness in North America. MSphere. 2020;5:e00056-20. https://doi.org/10.1128/ mSphere.00056-20

10. Aarestrup FM, Hendriksen RS, Lockett J, Gay K, Teates K, McDermott PF, et al. International spread of multidrugresistant Salmonella Schwarzengrund in food products. Emerg Infect Dis. 2007;13:726-31. https:/ / doi.org/10.3201/ eid1305.061489

11. Centers for Disease Control and Prevention. An atlas of Salmonella in the United States, 1968-2011 [cited 2021 Jun 22]. https:// www.cdc.gov/salmonella/pdf/ schwarzengrund-508c.pdf

12. Deblais L, Lorentz B, Scaria J, Nagaraja KV, Nisar M, Lauer D, et al. Comparative genomic studies of Salmonella Heidelberg isolated from chicken- and turkey-associated farm environmental samples. Front Microbiol. 2018;9:1841. https:// doi.org/10.3389/fmicb.2018.01841 
13. White PL, Green AL, Holt KG, Hale KR. Multidrug-resistant Salmonella enterica subspecies I serovar 4,[5],12:i:- isolates recovered from Food Safety and Inspection Service-regulated products and food animal ceca, 2007-2016. Foodborne Pathog Dis. 2019;16:679-86. https:/ / doi.org/10.1089/fpd.2018.2573

14. Routh JA, Pringle J, Mohr M, Bidol S, Arends K, AdamsCameron $\mathrm{M}$, et al. Nationwide outbreak of multidrug-resistant Salmonella Heidelberg infections associated with ground turkey: United States, 2011. Epidemiol Infect. 2015;143:322734. https://doi.org/10.1017/S0950268815000497
15. Mody RK, Meyer S, Trees E, White PL, Nguyen T, Sowadsky R, et al. Outbreak of Salmonella enterica serotype I 4,5,12:i:- infections: the challenges of hypothesis generation and microwave cooking. Epidemiol Infect. 2014;142:1050-60. https://doi.org/10.1017/S0950268813001787

Address for correspondence: Farrell A. Tobolowsky, Centers for Disease Control and Prevention, 1600 Clifton Rd NE, Mailstop H24-2, Atlanta, GA 30329-4027, USA; email: oqk3@cdc.gov

\section{January 2020 Viruses}

- Spatial Epidemiologic Trends and Hotspots of Leishmaniasis, Sri Lanka, 2001-2018

- Candidatus Mycoplasma haemohominis in Human, Japan

- Nutritional Care for Patients with Ebola Virus Disease

- Paid Leave and Access to Telework as Work Attendance Determinants during Acute Respiratory Illness, United States, 2017-2018

- Preclinical Detection of Prions in Blood of Nonhuman Primates Infected with Variant Creutzfeldt-Jakob Disease

- Effect of Acute Illness on Contact Patterns, Malawi, 2017

- Outbreak of Peste des Petits Ruminants among Critically Endangered Mongolian Saiga and Other Wild Ungulates, Mongolia, 2016-2017

- Elephant Endotheliotropic Herpesvirus Hemorrhagic Disease in Asian Elephant Calves in Logging Camps, Myanmar

- Risk Factors for and Seroprevalence of Tickborne Zoonotic Diseases among Livestock Owners, Kazakhstan

- High Azole Resistance in Aspergillus fumigatus Isolates from Strawberry Fields, China, 2018

- Tick-Borne Encephalitis Virus, United Kingdom

- Phenotypic and Genotypic Correlates of Penicillin Susceptibility in Nontoxigenic Corynebacterium diphtheriae, British Columbia, Canada, 2015-2018

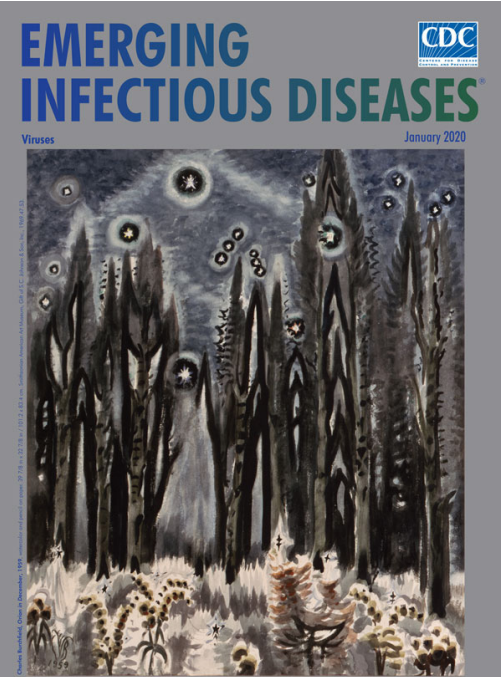

- High Pathogenicity of Nipah Virus from Pteropus lylei Fruit Bats, Cambodia

- Varicella in Adult Foreigners at a Referral Hospital, Central Tokyo, Japan, 2012-2016

- Geographic Distribution and Incidence of Melioidosis, Panama

- Shigella Bacteremia, Georgia, USA, 2002-2012

- Distribution of Japanese Encephalitis Virus, Japan and Southeast Asia, 2016-2018

- Novel Reassortant Highly Pathogenic Avian Influenza A(H5N2) Virus in Broiler Chickens, Egypt

- Infectivity of Norovirus GI and GII from Bottled Mineral Water during a Waterborne Outbreak, Spain
- Effect of Pediatric Influenza Vaccination on Antibiotic Resistance, England and Wales

- Locally Acquired Human Infection with Swine-Origin Influenza $A(H 3 N 2)$ Variant Virus, Australia, 2018

- Use of Ambulance Dispatch Calls for Surveillance of Severe Acute Respiratory Infections

- Hantavirus Pulmonary Syndrome in Traveler Returning from Nepal to Spain

- Visceral Leishmaniasis, Northern Somalia, 2013-2019

- Autochthonous Human Fascioliasis, Belgium

- Recombinant Nontypeable Genotype II Human Noroviruses in the Americas

- Legionella pneumophila as Cause of Severe Community-Acquired Pneumonia, China

- Training for Foodborne Outbreak Investigations by Using Structured Learning Experience

- Emergence of Vibrio cholerae 01 Sequence Type 75 in Taiwan

- Diabetes Mellitus, Hypertension, and Death among 32 Patients with MERS-CoV Infection, Saudi Arabia

- Influenza D Virus of New Phylogenetic Lineage, Japan

- Diagnosis of Syphilitic Bilateral Papillitis Mimicking Papilloedema

- Influenza A Virus Infections in Dromedary Camels, Nigeria and Ethiopia, 2015-2017 